\section{Seizures related to blood transfusion and erythropoietin treatment in patients undergoing dialysis}

\section{A L Brown, B Tucker, L R I Baker, A E G Raine}

\section{Department of}

Nephrology, St

Bartholomew's Hospital,

London EC1A 7BE

A L Brown, MRCP, research

fellow

B Tucker, MB, clinical

assistant

L R I Baker, MD, consultant nephrologist

A E G Raine, MRCP, consultant nephrologist

Correspondence to: Dr Brown.

BrMed f 1989;299:1258-9
Correction of the anaemia of chronic renal failure by recombinant human erythropoietin results in a rise in packed cell volume with a consequent increase in blood viscosity, predisposing to increased vascular resistance and the development of hypertension. ' Increases in blood pressure have occurred in almost half the patients treated so far with erythropoietin, ${ }^{1}$ but of more serious concern is the development of seizures in seven of these patients in the United Kingdom. ${ }^{2}$ Although these bear similarities to hypertensive encephalopathy, their mechanism remains unclear.

An episode of seizures associated with erythropoietin treatment in one of our patients has been reported.' We describe here a subsequent seizure suffered by this patient 11 days after a blood transfusion and two similar episodes suffered by an anephric patient undergoing continuous ambulatory peritoneal dialysis. These episodes are of interest because of their resemblance to the seizures reported in association with erythropoietin treatment.

\section{Case 1}

A 52 year old white woman with chronic glomerulonephritis had been maintained on haemodialysis since 1973. After her trial of erythropoietin ended in November 1987 she again became dependent on blood transfusions. After transfusion on 28 April 1988 her blood pressure increased from $140 / 75$ to $180 / 90 \mathrm{~mm} \mathrm{Hg}$ on 9 May with associated breathlessness and headaches. Her weight had increased by $3 \mathrm{~kg} ; 2.5 \mathrm{~kg}$ was removed through haemodialysis but the packed cell volume increased from $0 \cdot 18$ to 0.26 (figure). Her blood pressure rose, despite oral nifedipine, to $220 / 110 \mathrm{~mm}$ $\mathrm{Hg}$, when she suffered a grand mal fit. Blood pressure was subsequently controlled with intravenous hydralazine, then oral captopril. Plasma biochemical findings were stable, and serum glucose and calcium concentrations, fundoscopy, and a computed tomogram of the brain were all normal. At the time of writing the patient remained well on thrice weekly haemodialysis; blood pressure was $120 / 70 \mathrm{~mm} \mathrm{Hg}$ with oral captopril.

\section{Case 2}

A 31 year old Afro-Caribbean man with focal glomerulosclerosis underwent a renal transplant in 1979; this failed and he was maintained on haemodialysis. In 1981 bilateral nephrectomy was performed because of poorly controlled hypertension, and he became dependent on transfusions. He then transferred to continuous ambulatory peritoneal dialysis because of vascular access problems. On 23 September 1983, four weeks after uneventful transfusion (figure), he complained of headache, blurred vision, and vomiting. Blood pressure was $250 / 100 \mathrm{~mm} \mathrm{Hg}$. Two grand mal fits rapidly ensued. There were no signs of fluid overload, plasma biochemical findings were stable, and serum calcium and glucose concentrations and funduscopy, brain scan, and lumbar puncture results were normal. He was treated with oral hydralazine and propranolol and peritoneal dialysis. Four days later his blood pressure was $120 / 80 \mathrm{~mm} \mathrm{Hg}$ without antihypertensive medication.

On 10 December 1984 he had a three unit blood transfusion. Two days later he developed severe headache; he had gained $1.5 \mathrm{~kg}$. Blood pressure was $200 / 150 \mathrm{~mm} \mathrm{Hg}$. Funduscopy was normal and bio-
Time of seizures in relation to changes in packed cell volume and blood pressure with blood transfusions. BT= blood transfusion (number of units in parentheses)

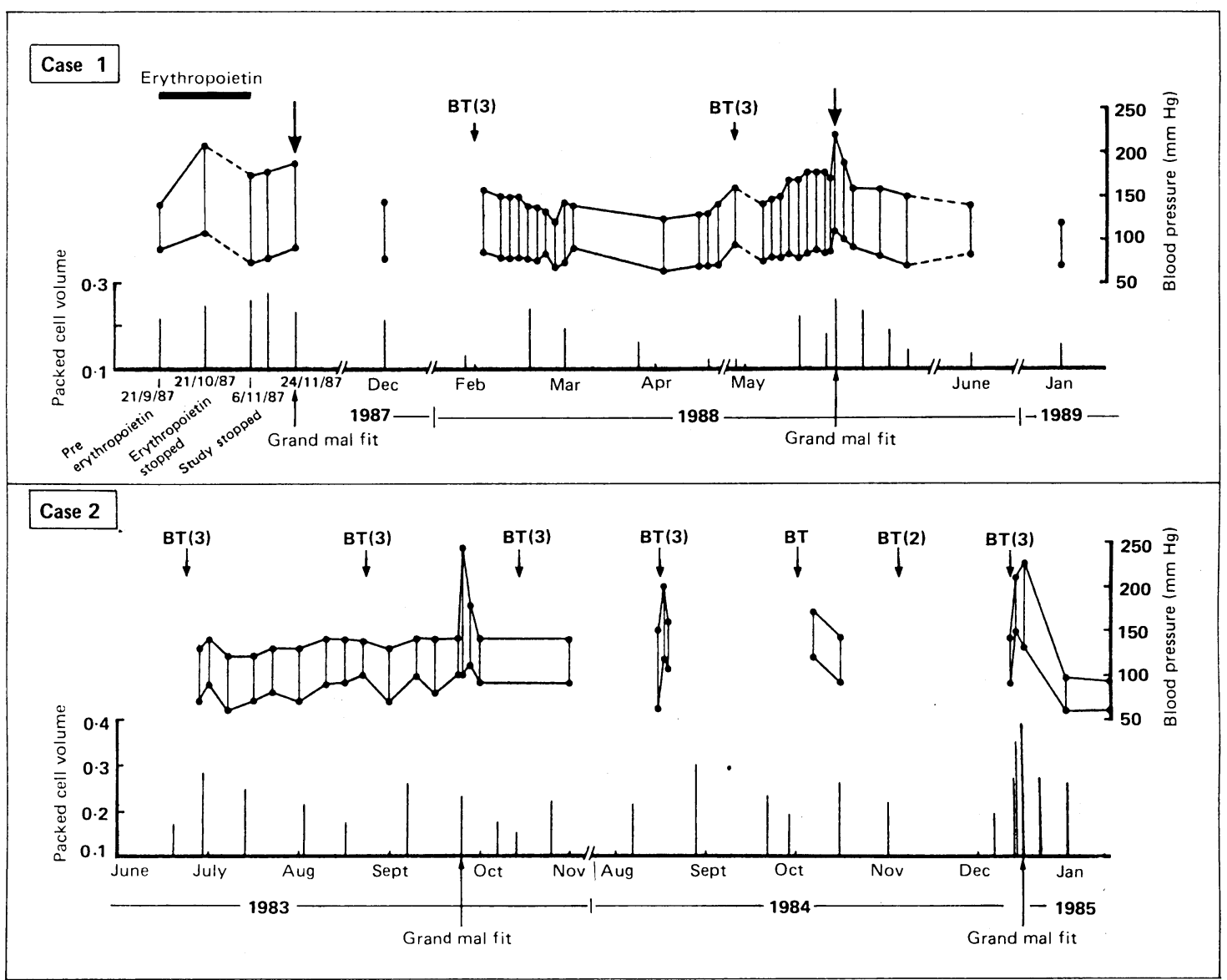


chemical findings stable. The packed cell volume was $0 \cdot 27$. With hypertonic peritoneal dialysis his weight decreased by $1.6 \mathrm{~kg}$, but blood pressure remained raised at $230 / 130 \mathrm{~mm} \mathrm{Hg}$, and the next day he suffered two generalised seizures. Packed cell volume was $0 \cdot 35$. Phenytoin was started. He was discharged on 17 December, normotensive with no neurological sequelae, and taking no antihypertensive medication. Phenytoin was subsequently withdrawn. He had a cadaveric renal transplant in 1987 and remained well with a blood pressure of $130 / 80 \mathrm{~mm} \mathrm{Hg}$.

\section{Comment}

Neither patient had previously suffered seizures. In both fitting occurred after a sudden rise in blood pressure in association with normal fundi and computed tomograms and in the context of recent blood transfusion and increase in packed cell volume. These features closely resemble those of seizures reported in patients treated with erythropoietin while undergoing dialysis. ${ }^{2}$ As with erythropoietin, the factors precipitat- ing fits in these patients remain unclear. In case 1 the haemoconcentration associated with haemodialysis may have been relevant. The second patient was not fluid overloaded at the time of the first seizure but was at the time of the second, when rapid fluid removal by peritoneal dialysis might have contributed. Perhaps a rapid rise in packed cell volume resulted in both increased blood viscosity and a loss of hypoxic vasodilatation ${ }^{3}$ and thus a rise in vascular resistance. These events, superimposed on a possibly abnormal cerebral vasculature, may have resulted in hypertensive encephalopathy. ${ }^{+}$

I Raine AEG. Hypertension, blood viscosity and cardiovascular morbidity in renal failure: implications of erythropoietin therapy. Lancet 1988;ii:97-9.

2 Edmunds ME, Walls J. Tucker B, $t$ al. Seizures in haemodialysis patients treated with recombinant human erythropoietin. Nephrology, Dialysis, Transplantation (in press).

3 Neff MS, Kim KE, Persoff M, Onesti G, Swartz C. Hemodynamics of uremic anemia. Circulation 1971:43:876-83.

4 Byrom FB. Pathogenesis of hypertensive encephalopathy and its relation to the malignant phase of hypertension: experimental evidence from the hypertensive rat. Lancet 1954;ii:201-11.

(Accepted 25 August 1989)

\section{Hay fever, hygiene, and household size}

\section{David P Strachan}

\section{Department of \\ Epidemiology and Population Sciences, London School of Hygiene and Tropical Medicine, London WC1E 7HT \\ David P Strachan, MRCP, lecturer in epidemiology}

Br Med f 1989;299:1259-60
Hay fever has been described as a "post industrial revolution epidemic," and successive morbidity surveys from British general practice suggest that its prevalence has continued to increase over the past 30 years. ${ }^{2}$ Other evidence suggests a recent increase in the prevalence of asthma ${ }^{2}$ and childhood eczema. ${ }^{3}$ This paper suggests a possible explanation for these trends over time.

\section{Subjects, methods, and results}

I studied the epidemiology of hay fever in a national sample of 17414 British children born during one week in March 1958 and followed up to the age of 23 years (the National Child Development Study). Three outcomes were investigated: $(a)$ self reported "hay fever during the past 12 months" at age $23 ;(b)$ parental report of "hay fever or allergic rhinitis in the past 12 months" at age $11 ;(c)$ parental recall of "eczema in the first year of life" elicited when the child was 7 . Cross tabulations were performed with the SAS statistical package, and multiple logistic regression models were fitted with the LR program in the BMDP statistical package.

Of the 16 perinatal, social, and environmental factors studied the most striking associations with hay fever were those for family size and position in the household in childhood. The table shows that at both 11 and 23 years of age hay fever was inversely related to the number of children in the household at age 11 (when it is assumed most families were complete). When prevalence figures were adjusted by multiple logistic regression for other significant determinants of hay fever in this cohort (see table) the associations with numbers of older and younger children in the household persisted. These trends in adjusted prevalence were independent of one another and each was significant $(\mathrm{p}<0.01$, see table $)$, but the trends by number of older children were significantly steeper $\left(\chi^{2}=11 \cdot 6, \mathrm{df}=1\right.$, $\mathrm{p}<0.01$ at age $11 ; \chi^{2}=19.5, \mathrm{df}=1, \mathrm{p}<0.01$ at age 23). A further analysis of hay fever occurring at 23 by birth

Prevalence of hay fever and of eczema in infancy by position in the household. Numbers in parentheses

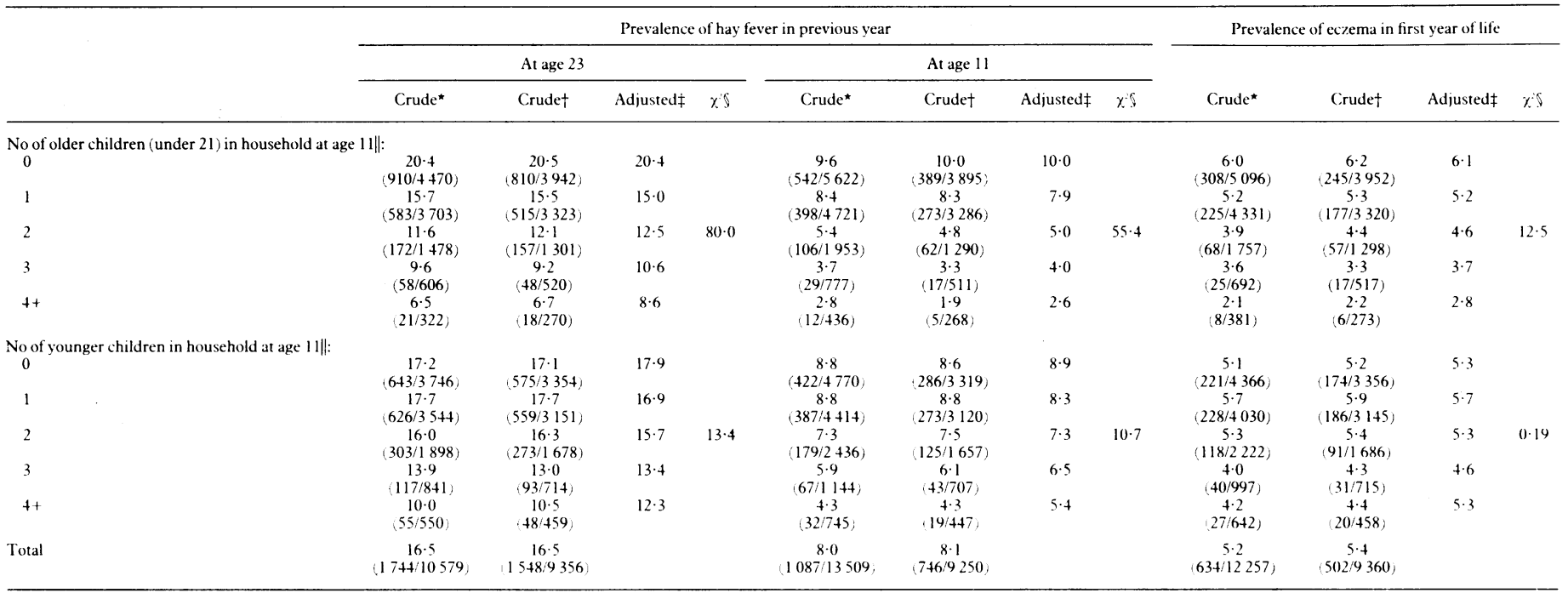

*Using all available information

FFor subjects with complete covariate data included in the multiple logistic regression.

tAdjusted by multiple logistic regression for the other factor in the table, plus father's social class, housing tenure and shared household amenities in childhoxd, breast feeding, region of birth, and cigarette smoking at 23. Test for linear trend $(\mathrm{df}=1)$ from the multiple logistic regression model.

Includes children of the family living away from home in 1969. 